Experiments on the Scaling of Ionization Balance vs. Electron and Radiation Temperature in Non-LTE Gold Plasmas

R. F. Heeter, S. B. Hansen, P. Beiersdorfer, M. E.

Foord, K. B. Fournier, D. H. Froula, A. J. Mackinnon, M. J. May, M. B. Schneider, B. K. F. Young

July 9,2004

14th APS Topical Conference on Atomic Processes in Plasmas

Santa Fe, NM, United States

April 19, 2004 through April 22, 2004 
This document was prepared as an account of work sponsored by an agency of the United States Government. Neither the United States Government nor the University of California nor any of their employees, makes any warranty, express or implied, or assumes any legal liability or responsibility for the accuracy, completeness, or usefulness of any information, apparatus, product, or process disclosed, or represents that its use would not infringe privately owned rights. Reference herein to any specific commercial product, process, or service by trade name, trademark, manufacturer, or otherwise, does not necessarily constitute or imply its endorsement, recommendation, or favoring by the United States Government or the University of California. The views and opinions of authors expressed herein do not necessarily state or reflect those of the United States Government or the University of California, and shall not be used for advertising or product endorsement purposes. 


\title{
Experiments on the Scaling of Ionization Balance vs. Electron and Radiation Temperature in Non-LTE Gold Plasmas
}

\author{
R.F. Heeter, S.B. Hansen, P. Beiersdorfer, M.E. Foord, K.B. Fournier, D.H. \\ Froula, A.J. Mackinnon, M.J. May, M.B. Schneider, B.K.F. Young
}

Lawrence Livermore National Laboratory; 7000 East Avenue, L-472; Livermore, CA 94550

\begin{abstract}
.
Understanding and predicting the behavior of high-Z non-LTE plasmas is important for developing indirect-drive inertial confinement fusion. Extending earlier work from the Nova laser, we present results from experiments using the Omega laser to study the ionization balance of gold as a function of electron and radiation temperature. In these experiments, gold samples embedded in Be disks expand under direct laser heating to $n_{e} \approx 10^{21} \mathrm{~cm}^{-3}$, with $T_{e}$ varying from 0.8 to $2.5 \mathrm{keV}$. An additional finite radiation field with effective temperature $T_{r}$ up to $150 \mathrm{eV}$ is provided by placing the gold-Be disks inside truncated $1.2 \mathrm{~mm}$ diameter tungsten-coated cylindrical hohlraums with full laser entrance holes. Densities are measured by imaging of plasma expansion. Electron temperatures are diagnosed with either $2 \omega$ or $4 \omega$ Thomson scattering, and also K-shell spectroscopy of $\mathrm{KCl}$ tracers co-mixed with the gold. Hohlraum flux and effective radiation temperature are measured using an absolutely-calibrated multichannel filtered diode array. Spectroscopic measurements of the M-shell gold emission in the 2.9-4 keV spectral range provide ionization balance and charge state distribution information. The spectra show strong variation with $T_{e}$, strong variation with the applied $T_{r}$ at $T_{e}$ below $1.6 \mathrm{keV}$, and relatively little variation with $T_{r}$ at higher $T_{e}$ (upwards of $2 \mathrm{keV}$ ). We summarize our most recent spectral analyses and discuss emerging and outstanding issues.
\end{abstract}

\section{INTRODUCTION}

Accurate prediction of target behavior in indirect-drive inertial confinement fusion (ICF) requires a detailed understanding of the driver coupling to the hohlraum and the subsequent coupling of the hohlraum radiation to the capsule [1]. Both processes involve the low-density, non-LTE plasma which blows off from the gold hohlraum walls. To understand the X-ray absorption and emission, one must know the ionization balance and charge state distribution of these plasmas under relevant conditions. For laserdriven hohlraums there is particular interest in the physics below the critical density $n_{e}<n_{c} \approx 10^{22} \mathrm{~cm}^{-3}$. There is also special interest in the M-shell ionization states of gold because the M-band X-ray emission can cause capsule preheat problems.

Code comparisons and experimental data have begun to illuminate this topic. A mid1990s comparison of 9 different model predictions of the mean ionization $\langle Z\rangle$ versus electron temperature $0.8<T_{e}<2.8 \mathrm{keV}$ for gold plasmas at $n_{e}=10^{20} \mathrm{~cm}^{-3}$ found a total spread among the models of 14-19 charge states out of a mean $\langle\mathrm{Z}\rangle$ ranging from $\sim 40$ to $\sim 55$ [2]. Benchmark experiments were performed in the late 1990s at the Nova laser. These used laser-heated, Be-tamped Au foils at $n_{e}=6 \times 10^{20} \mathrm{~cm}^{-3} \pm 20 \%$ and 
$T_{e}=2.2 \mathrm{keV} \pm 10 \%$, and analyzed the $\mathrm{n}=5$ to $\mathrm{n}=3$ transitions between 3.3 and $3.5 \mathrm{keV}$, from which $\langle Z\rangle=49.3 \pm 0.5$ was inferred [3, 4]. No hohlraum was used (radiation temperature $T_{r}<50 \mathrm{eV}$ ). Other experiments characterized the hohlraum blowoff directly, in conditions with finite $T_{r}$ but also density and temperature gradients [5]. More recently, the gold ionization balance was studied in a low-density coronal equilibrium $\left(n_{e} \approx\right.$ $10^{12} \mathrm{~cm}^{-3}$ ) [6]. Finally, a new round of code comparisons showed better agreement near the Nova $2.2 \mathrm{keV}$ data point [7]. However, the coronal data agree poorly with the models, and at higher densities experiments have yet to test the effects of varying electron and radiation temperature. The challenge for ICF target-design radiation-hydrodynamics codes is further increased because they need computationally cheap non-LTE models.

As a next step in this area, we have conducted the first systematic experimental study of the ionization balance and charge state distribution in a well-characterized high- $Z$ material as a function of both the electron and radiation temperature [8]. We have used the Omega laser to replicate the Nova conditions, and then studied the Au 5-3 emission over a broad electron temperature range (1-3 keV), with effective radiation temperatures from $T_{r}<50 \mathrm{eV}$ to $T_{r} \approx 150 \mathrm{eV}$.

\section{EXPERIMENTAL CONFIGURATION}

The experimental details have been discussed elsewhere [8], so here we provide only a synopsis. The targets were Be disks, $11 \mu \mathrm{m}$ thick and 300-400 $\mu \mathrm{m}$ in diameter, containing a thin $200 \mu \mathrm{m}$ diameter embedded layer of either $\mathrm{Au}$ or co-mixed $\mathrm{Au}: \mathrm{KCl}$. About half of the targets were placed inside tungsten-coated hohlraums $(1.2 \mathrm{~mm}$ in diameter, $1.6 \mathrm{~mm}$ in length, with fully open laser entrance holes). With these hohlraums, the geometry at Omega allows up to 6 beams of $3 \omega$ light to hit each side of the disk directly $\left(23.2^{\circ}\right.$ at $f / 6.7$, i.e. $\left.\pm 4.3^{\circ}\right)$, while up to 30 beams heat the hohlraum walls (at $47.8^{\circ}$ and $60^{\circ}$ ).

Figure 1 shows how the experiment works. All beams used 2 ns square pulses. One or two beams on each side fire early to preheat the disk, burn through the Be and expand the $\mathrm{Au}$. One, two or four beams on each side then heat the disk to a desired $T_{e}$, with intensity on the sample of $I=1.7-7.0 \times 10^{14} \mathrm{~W} / \mathrm{cm}^{2}$. During this latter pulse the hohlraum (if present) is also heated. The $2 \omega$ or $4 \omega$ probe beam runs from $+1.5 \mathrm{~ns}$ to $+3.5 \mathrm{~ns}$.

Gated pinhole cameras with axial and quasi-perpendicular $\left(79.2^{\circ}\right)$ views through slots in the hohlraum provide information on the Au disk's expansion (density). An absolutely-calibrated filtered X-ray diode array (Dante) measures the hohlraum X-ray flux over 10 spectral bands, yielding the effective $T_{r}$ [9]. Another framing camera at $79.2^{\circ}$ uses a convex cylindrically-bent RAP crystal spectrometer (TSPEC)[10] to measure the Au: $\mathrm{KCl}$ sample's emission through slots in the hohlraum. The TSPEC's 80 $\mu \mathrm{m}$ entrance slit spatially resolves the sample's emission from the hohlraum's emission. Finally, scattered light from the $2 \omega$ (or $4 \omega$ ) probe beam travels to an optical (or UV) spectrometer, which records the Thomson scattering (TS) spectrum, which is sensitive to $T_{e}$, using the method described in the Nova experiments $[3,5]$. 


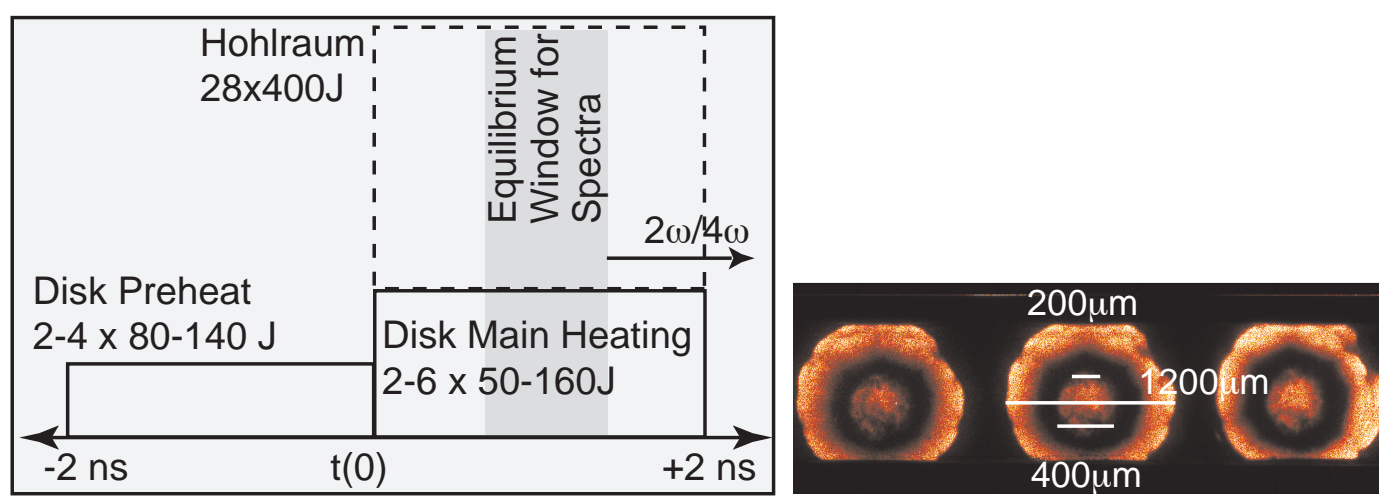

FIGURE 1. Left: Laser timing diagram, with disk preheat and main heating, hohlraum heating and probe beams, plus equilibrium window of interest for spectroscopy. Right: Pinhole images of disk in hohlraum taken by X-ray framing camera at $t \approx 0.3 \mathrm{~ns}$. Bars show diameters of hohlraum $(1200 \mu \mathrm{m})$, disk $(400 \mu \mathrm{m})$ and buried Au layer $(200 \mu \mathrm{m})$. Time interval between images is $80 \mathrm{ps}$.

\section{PLASMA CONDITIONS}

From the Nova experiments and simulations [4], one expects the expanding sample to reach conditions of interest by $t=0.7 \mathrm{~ns}$ [3]. There was no measurable radial expansion of the sample, so the sample density is determined from the axial expansion relative to the initial areal density. We measure the axial expansion to be $270 \mu \mathrm{m}$ at the time of interest. The sample had an initial areal density of $145 \mu \mathrm{g} / \mathrm{cm}^{2}$ of $\mathrm{Au}$ and $37 \mu \mathrm{g} / \mathrm{cm}^{2}$ of $\mathrm{KCl}$. We use $\langle\mathrm{Z}\rangle=45$ for $\mathrm{Au}$ and 17 for $\mathrm{KCl}$ and conclude that $n_{e}=1.1 \times 10^{21} \mathrm{~cm}^{-3}$. We estimate $20 \%$ shot-to-shot variation in $n_{e}$ and $30 \%$ overall uncertainty. These densities are 1.8 times higher than the Nova data, but the ionization balance in this regime is not strongly dependent on density, so the data should be comparable. These experiments also meet the ionization-equilibrium conditions of the Nova experiments [3].

For targets with hohlraums, the radiation temperature was measured by the Dante to be $175 \mathrm{eV} \pm 5 \%$ during the time of interest. Because these hohlraums have both wide-open laser entrance holes and multiple diagnostic slots, the hohlraum wall only covers $61 \%$ of the solid angle seen by the sample. The X-ray flux on the sample is correspondingly reduced from what would be seen if the sky were $175 \mathrm{eV}$ in all directions, so the effective $T_{r}=155 \mathrm{eV}$. The M-band channel on the Dante (filtered for X-rays $>\sim 2 \mathrm{keV}$ ) contributes up to $18 \%$ of the total flux during the time range of interest.

Two initial attempts to record TS spectra from samples within hohlraums were unsuccessful, but the $2 \omega$ and $4 \omega$ TS systems (commissioned at Omega in 2002 and 2003, respectively) provided $T_{e}$ data for the plain-disk targets. The TS beam was fired after the density and spectroscopic data were acquired, mitigating any perturbative effects of the probe. Under similar conditions the Nova and Omega TS data agree to within their $10-20 \%$ error bars. The Omega TS data is roughly consistent with a $T_{e} \sim I^{2 / 3}$ scaling expected theoretically [1, p.3995]. Fitting the TS data to this scaling allows the temperature to be estimated in all shots (even those without TS data). From this we infer $T_{e}=0.8$ $\mathrm{keV}$ at the low end of our intensity range, $I=1.4 \times 10^{14} \mathrm{~W} / \mathrm{cm}^{2}$. 


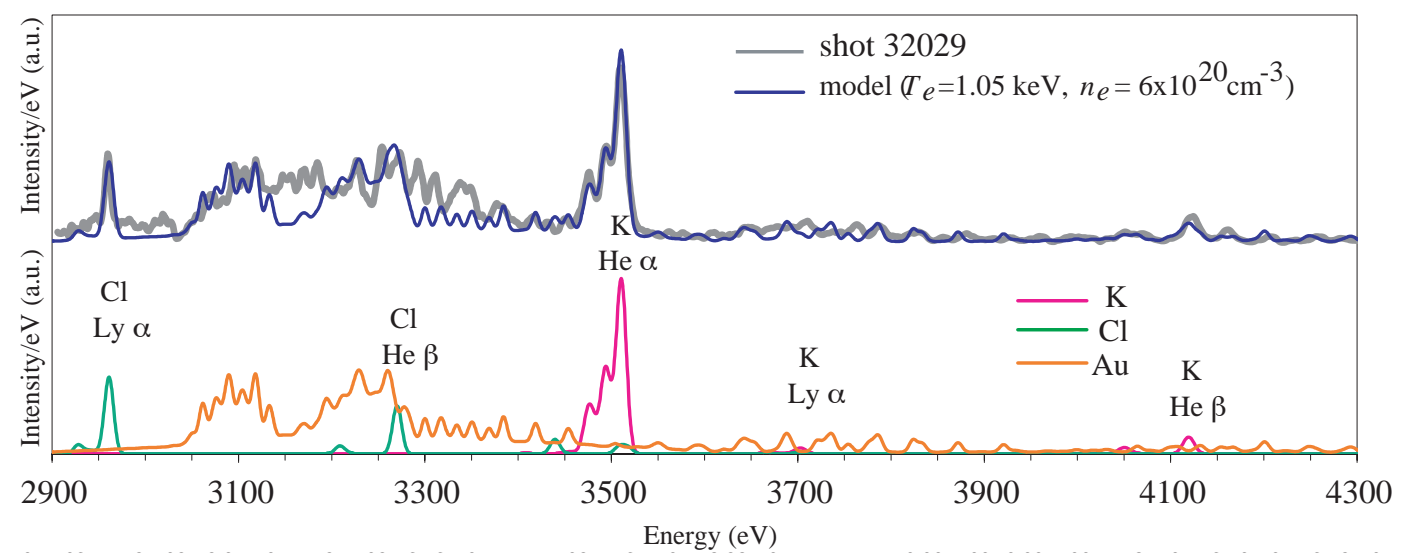

FIGURE 2. Top: Experimental Au: $\mathrm{KCl}$ emission at $t=0.9 \mathrm{~ns}$ from disk heated at $2.9 \times 10^{14} \mathrm{~W} / \mathrm{cm}^{2}$, and theoretical model spectrum from FAC. Bottom: contributions to model spectrum from each of the elements present.

The sample temperature can also be determined by fitting the K-shell emission spectra from the KCL tracers in the sample. We will discuss the $\mathrm{Au}: \mathrm{KCl}$ spectra in detail below, but we note here that at the higher, Nova-equivalent intensities, the $\mathrm{KCl} \mathrm{K}$-shell fits give $T_{e}$ roughly $40 \%$ lower than the TS data, and the error bars do not overlap. At the lower end of our intensity range, the K-shell fits are still below the TS data, but within the combined error bars. Further work is needed to understand this.

\section{GOLD EMISSION SPECTRA}

The TSPEC snout mounted on a standard Omega X-ray framing camera produced 4 gated spectra per shot, with spectral resolving power $E / \Delta E>100$ in the spectral band of greatest interest ( 2.9 to $3.5 \mathrm{keV}$ ). More than 30 spectra of acceptable quality have been obtained, from more than 15 shots, with timings between $\mathrm{t}=0.5 \mathrm{~ns}$ (when the sample reaches conditions of interest) and $t=1.5 \mathrm{~ns}$ (when the TS probe beam turns on). The data for disk-only targets span the laser intensity range from 1.4 to $7.0 \times 10^{14} \mathrm{~W} / \mathrm{cm}^{2}$, with $T_{e}$ ranging from 0.8 to $2.5 \mathrm{keV}$, scaling from the TS data. The comparable ranges for disk-in-hohlraum targets are $I=1.4$ to $5.2 \times 10^{14} \mathrm{~W} / \mathrm{cm}^{2}$ and $T_{e}$ from 0.8 to $2.1 \mathrm{keV}$ (not including the effects of the hohlraum radiation field).

Fig. 2 shows one spectrum from a relatively cold disk shot at $I=2.9 \times 10^{14}\left(T_{e} \approx 1.4\right.$ $\mathrm{keV}$ based on TS), together with the results of modeling the $\mathrm{Au}, \mathrm{K}$, and $\mathrm{Cl}$ separately and then summing the results. The model (using the Flexible Atomic Code, FAC [11]) includes neutral to bare ions for all 3 elements. For $\mathrm{K}$ and $\mathrm{Cl}$ the 1:1 KCL stoichiometry is imposed, and $\sim 500$ levels are used ( $n \leq 5+$ autoionizing states for $\mathrm{H}, \mathrm{He}, \mathrm{Li}$ and Be-like ions). For the $\mathrm{Au}, \sim 30,000$ levels are used ( $n \leq 6+$ autoionizing, for Fe-like $(+53)$ through $\mathrm{Kr}$-like (+43) ions). As one would hope, the fits for $\mathrm{KCl}$ are quite good, including the structure of the K He- $\alpha$ complex; however, the fit yields $T_{e}=1.0 \mathrm{keV}$. Increasing $T_{e}$ to match the TS data causes the $\mathrm{K} \mathrm{Ly}-\alpha$ line to grow excessively. 


\section{DISCUSSION}

Although much data remain to be analyzed, it is possible to draw some useful conclusions. The Omega and Nova spectra look similar under comparable conditions, providing further support for models which agree with the Nova data. However, the discrepancy between the temperatures inferred from $\mathrm{KCl} \mathrm{K}$-shell emission and Thomson scattering must be resolved. Further work here includes both verifying the relative calibration of the X-ray spectra, and cross-comparing different K-shell models. It would also help to repeat the shots with improvements in both the X-ray and Thomson spectrometers. Meanwhile, the dominant Au lines in the lower-temperature Omega data (Fig. 2) are quite different from those in the published Nova data. The fit in Fig. 2 yields $\langle\mathrm{Z}\rangle=47$ at $T_{e}=1.05-1.4 \mathrm{keV}$, two charge states down from the Nova data at $2.2 \mathrm{keV}$. Further work is needed to establish the error bars on the Omega $\langle\mathrm{Z}\rangle$ analysis. We conclude by noting that the Omega spectra also vary with $T_{r}$, but due to space constraints this topic is deferred to a future publication.

\section{ACKNOWLEDGMENTS}

The authors thank R.W. Lee, B. Wilson and C.A. Back for comments and information, A. Osterheld and P. Springer for project support, V. Rekow, C. Sorce, and K. Loughman for diagnostic support, and the Omega crew for successful shot execution. This work

was performed under the auspices of the U.S. Department of Energy by University of California, Lawrence Livermore National Laboratory under Contract W-7405-Eng-48.

\section{REFERENCES}

1. Lindl, J., Phys. Plasmas, 2, 3933-4024 (1995).

2. Lee, R. W., Nash, J. K., and Ralchenko, Y., J. Quant. Spect. Rad. Transfer, 58, 737 (1997).

3. Foord, M. E., Glenzer, S. H., Thoe, R. S., Wong, K. L., Fournier, K. B., Wilson, B. G., and Springer, P. T., Phys. Rev. Lett. , 85, 992 (2000).

4. Foord, M. E., Glenzer, S. H., Thoe, R. S., Wong, K. L., Fournier, K. B., Albritton, J. R., Wilson, B. G., and Springer, P. T., J. Quant. Spect. Rad. Transfer, 65, 231 (2000).

5. Glenzer, S. H., Fournier, K. B., Wilson, B. G., Lee, R. W., and Suter, L. J., Phys. Rev. Lett. , 87 (2001).

6. Wong, K. L., May, M. J., Beiersdorfer, P., Fournier, K. B., Wilson, B. G., Brown, G. V., Springer, P. T., Neill, P. A., and Harris, C. L., Phys. Rev. Lett. , 90, 235001 (2003).

7. Bowen, C., Decoster, A., Fontes, C. J., Fournier, K. B., Peyrusse, O., and Ralchenko, Y. V., J. Quant. Spect. Rad. Transfer, 81, 71 (2003).

8. Heeter, R. F., Foord, M. E., Fournier, K. B., Froula, D. H., MacKinnon, A. J., May, M. J., Schneider, M. B., and Young, B. K. F., Fusion Sci. \& Tech. (2004), to be published.

9. Kornblum, H. N., Kauffman, R. L., and Smith, J. A., Rev. Sci. Instrum., 57, 2179 (1986).

10. Back, C. A., Kauffman, R. L., Bell, P. M., and Kilkenny, J. D., Rev. Sci. Instrum., 66, 764 (1995).

11. Gu, M.-F., Astrophys. J. , 589, 1085-1088 (2003). 